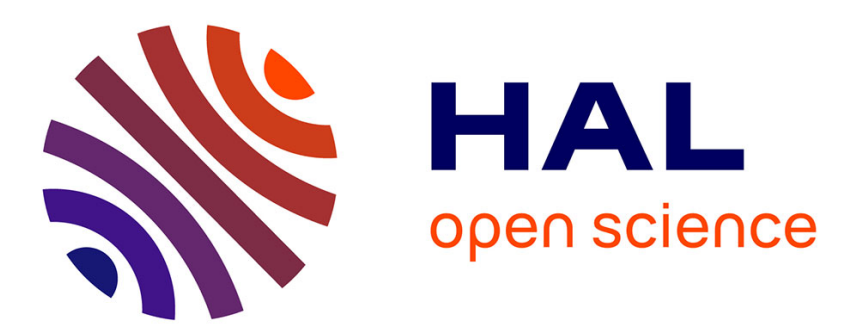

\title{
Using Cluster Dynamics to Model Electrical Resistivity Measurements in Precipitating Al-Sc Alloys
}

\author{
Emmanuel Clouet, Alain Barbu
}

\section{To cite this version:}

Emmanuel Clouet, Alain Barbu. Using Cluster Dynamics to Model Electrical Resistivity Measurements in Precipitating Al-Sc Alloys. Acta Materialia, 2007, 55, pp.391-400. 10.1016/j.actamat.2006.08.021 . hal-00115193

\section{HAL Id: hal-00115193 \\ https://hal.science/hal-00115193}

Submitted on 20 Nov 2006

HAL is a multi-disciplinary open access archive for the deposit and dissemination of scientific research documents, whether they are published or not. The documents may come from teaching and research institutions in France or abroad, or from public or private research centers.
L'archive ouverte pluridisciplinaire HAL, est destinée au dépôt et à la diffusion de documents scientifiques de niveau recherche, publiés ou non, émanant des établissements d'enseignement et de recherche français ou étrangers, des laboratoires publics ou privés. 


\title{
Using cluster dynamics to model electrical resistivity measurements in precipitating Al-Sc alloys
}

\author{
Emmanuel Clouet ${ }^{*}$ and Alain Barbu \\ Service de Recherches de Métallurgie Physique, CEA/Saclay, \\ 91191 Gif-sur-Yvette, France
}

\begin{abstract}
Electrical resistivity evolution during precipitation in Al-Sc alloys is modeled using cluster dynamics. This mesoscopic modeling has already been shown to correctly predict the time evolution of the precipitate size distribution. In this work, we show that it leads too to resistivity predictions in quantitative agreement with experimental data. We only assume that all clusters contribute to the resistivity and that each cluster contribution is proportional to its area. One interesting result is that the resistivity excess observed during coarsening mainly arises from large clusters and not really from the solid solution. As a consequence, one cannot assume that resistivity asymptotic behavior obeys a simple power law as predicted by LSW theory for the solid solution supersaturation. This forbids any derivation of the precipitate interface free energy or of the solute diffusion coefficient from resistivity experimental data in a phase-separating system like Al-Sc supersaturated alloys.
\end{abstract}

Key words: Precipitation, Resistivity, Kinetics, Aluminum alloys, Cluster dynamics

PACS: 64.60.Cn, 64.60.-i, 64.70.Kb, 64.75.+g

\section{Introduction}

Resistivity measurements are usually a convenient way to follow precipitation kinetics in phase-separating systems. Indeed, assuming that resistivity is proportional to the solid solution solute content as it is usually done [1], one gets direct access to the solute supersaturation. The so-called LSW theory (Lifshitz and Slyozov [2] and Wagner [3]) as extended by Ardell [4] allows then

* emmanuel.clouet@cea.fr 
to deduce from these measurements key parameters like the solubility, the precipitate interface free energy and the solute diffusion coefficient.

Numerous such measurements exist in Al-Sc alloys [5-10]. They have been intended to characterize the precipitation of the $\mathrm{Al}_{3} \mathrm{Sc} \mathrm{L}_{2}$ structure in aluminum alloys. In a previous study [11], we used cluster dynamics to model precipitation in $\mathrm{Al}-\mathrm{Zr}$ and Al-Sc alloys. This mesoscopic modeling was shown to provide predictions of the precipitate size distributions in quantitative agreement with available experimental data [12-14]. It is therefore worth seeing if such an agreement can be obtained also with resistivity measurements. The purpose is mainly to see how predictive cluster dynamics can be, i.e. if one can obtain reliable information concerning the precipitates as well as the solid solution in the whole time range of precipitation kinetics and not only in the asymptotic limit of the coarsening stage like LSW theory does. Confronting cluster dynamics predictions with resistivity measurements will validate too the multiscale approach developed for precipitation in Al-Zr-Sc alloys $[11,15-18]$. The use of resistivity experiments to validate a multiscale kinetic modeling has already proved its efficiency for irradiated iron [19].

The first part of this article explains how resistivity can be modeled within the framework of cluster dynamics. A thorough comparison between the obtained model predictions and available experimental data is then performed. This allows us to conclude on the validity of the interface free energies which are obtained through such measurements.

\section{Cluster gas model of electrical resistivity}

Cluster dynamics models the phase-separating alloy as a gas of solute clusters which exchange solute atoms by single atom diffusion. Clusters are assumed to be spherical and are described by a single parameter, the number $n_{\mathrm{Sc}}$ of solute atoms they contain. The time evolution of the cluster size distribution is governed by a master equation which input parameters are the solute diffusion coefficient and the precipitate interface free energy. For Al-Sc alloys, these needed parameters were directly deduced from an atomic diffusion model $[15$, 16,18] previously built for Al-Zr-Sc alloys. It should be stressed that, although none of these input parameters were intentionally fitted, cluster dynamics managed to reproduce reasonably experimental data on the time evolution of the precipitate size distribution. The reader is referred to Ref. 11 for a full description of cluster dynamics modeling and its application to Al-Sc alloys. We will only describe in the following how this technique can be adapted to model electrical resistivity of a phase-separating Al-Sc alloy. 


\subsection{Cluster contribution to electrical resistivity}

So as to use cluster dynamics to simulate the evolution of the electrical resistivity during the annealing of a supersaturated solid solution, we assume that the total resistivity of the cluster gas is given by the sum of each cluster resistivity. Doing so, we neglect any interference between clusters. Therefore, the input parameters of the modeling are the contributions of each cluster, i.e. $\rho_{n_{\mathrm{Sc}}}$ for a cluster containing $n_{\mathrm{Sc}}$ solute atoms. Some calculations of this contribution exist in the literature (for a review, see Ref. 1). Most of them have been aimed to explain the increase of resistivity at the beginning of the precipitation kinetics in alloys like $\mathrm{Al}(\mathrm{Zn}), \mathrm{Al}(\mathrm{Ag}), \mathrm{Al}(\mathrm{Cu})$ or $\mathrm{Al}(\mathrm{Cr})$, showing that this resistivity increase is associated with the apparition of small clusters $(\sim 10-20 \AA)$.

All these calculations consider the elastic scattering of valence electrons by the perturbing potential due to the presence of the solute atoms composing the cluster. They show that solute atoms which are agglomerated in a small cluster can have a higher resistivity than the same isolated solute atoms because of Bragg scattering. Indeed, the smallest the cluster, the most relaxed the Bragg conditions for the electron scattering by the perturbing potential. This can lead to a high resistivity for small clusters. The scattering anisotropy increases with the cluster size and, as the cluster size gets of the order of the electron mean free path, the scattering becomes Bragg-like leading to a small contribution of the cluster to the resistivity.

In Al-Sc alloys, such an increase of the resistivity in the beginning of the precipitation kinetics has never been observed experimentally [5-10]. Actually, it does not seem to exist in alloys where the solute is a transition element and the solvent a free electron like metal [20]. Therefore, precise calculations of each cluster contribution to the electrical resistivity may not be required for Al-Sc alloys. Moreover, these calculations are limited to small clusters as one assumes that the perturbing potential of the cluster does not modify the Fermi surface of the solvent and that electrons are scattered only once by the cluster (Born approximation). Such assumptions are reasonable only as long as the cluster does not contain more than a few solute atoms $(\sim 10)$ but do not really apply for larger clusters. On the other hand, for clusters with a stoichiometric composition, one can consider that the conductivity is infinite inside the cluster and that electrons are only scattered by the interface between the cluster and the matrix. For a sharp interface, this leads to a cluster contribution to the resistivity proportional to its cross section [1] and therefore to its interface area as clusters are assumed spherical. This leads then to $\rho_{n_{\mathrm{Sc}}}=\rho_{1} n_{\mathrm{Sc}}{ }^{2 / 3}$, and the electrical resistivity of the phase separating system at time $t$ is simply 
given by

$$
\rho(t)=\rho_{\mathrm{Al}}^{0}+\rho_{1} \sum_{n_{\mathrm{Sc}}=1}^{n_{\mathrm{Sc}}^{*}} C_{n_{\mathrm{Sc}}}(t) n_{\mathrm{Sc}}{ }^{2 / 3}
$$

where $C_{n_{\mathrm{Sc}}}(t)$ is the atomic fraction of clusters containing $n_{\mathrm{Sc}}$ solute atoms. The time evolution of this cluster size distribution is governed by the cluster dynamics master equations (Eq. 2 and 3 in Ref. 11). In Eq. 1, we have assumed that only clusters smaller than a critical size $n_{\mathrm{Sc}}^{*}$ contribute to the electrical resistivity. We will see below which value this critical size has to be given in order to fit experimental data. $\rho_{\mathrm{Al}}^{0}$ is the electrical resistivity of the solvent (Al in this case). It is temperature dependent and is known experimentally. The only remaining unknown parameter in Eq. 1 is the contribution $\rho_{1}$ of a solute monomer to the electrical resistivity.

The increase of resistivity with Sc content has been measured at $77 \mathrm{~K}$ by Fujikawa et al. [6,21] who give $\delta \rho_{\mathrm{Sc}}=3400 \mathrm{n} \Omega \mathrm{m}$ per Sc atomic fraction. These measurements have been performed in under-saturated and therefore very dilute Al-Sc solid solutions. One can reasonably assumes that most of the solute are monomers and ignore larger clusters ${ }^{1}$, with the consequence that $\rho_{1}=\delta \rho_{\mathrm{Sc}}=3400 \mathrm{n} \Omega \mathrm{m}$. Assuming that Matthienssen rule [1] is obeyed, this quantity does not depend on the temperature. Different measured values of $\delta \rho_{\mathrm{Sc}}$ ranging from 3000 to $8200 \mathrm{n} \Omega \mathrm{m}$ per Sc atomic fraction can be found in the literature (for a review, see Tab. 2 in Ref. 10). Nevertheless we find that the value measured by Fujikawa et al. $[6,21]$ is the one that leads to the best agreement between the simulated and the experimental resistivity variations during precipitation kinetics.

\subsection{Critical size}

As already recalled above, the input parameters of cluster dynamics, i.e. the precipitate interface free energy and the Sc diffusion coefficients, were directly deduced from an atomic diffusion model [11]. The only left parameter needed to calculate electrical resistivity is the critical size $n_{\mathrm{Sc}}^{*}$ appearing in Eq. 1. So as to determine this boundary between clusters which are contributing or not to the electrical resistivity, we compare our predictions using different values for $n_{\mathrm{Sc}}^{*}$ with experimental data (Fig. 1). If one assumes that only monomers are contributing to the electrical resistivity $\left(n_{\mathrm{Sc}}^{*}=1\right)$, cluster dynamics do not reproduce experimental data. Indeed the simulated resistivity decreases too quickly during the precipitation kinetics. So as to obtain the plateau experimentally observed at the beginning, one has to take into account the con-

1 The maximal solubility limit of Sc in aluminum is 0.288 at.\%. We have checked with our cluster gas model of electrical resistivity in Al-Sc that only the monomer contribution is relevant for such a low nominal concentration. 
tributions of small clusters $\left(n_{\mathrm{Sc}} \leq 10\right)$, especially the dimer one. With these contributions, the simulated resistivity decreases at the right time, but the final plateau experimentally observed is not reproduced. Indeed, cluster dynamics predicts that resistivity decreases to reach a value close to the one corresponding to the equilibrium solid solution, whereas experimental data show an excess resistivity. The final plateau corresponding to this excess resistivity can only be obtained if one considers that all clusters are contributing to the resistivity $\left(n_{\mathrm{Sc}}^{*}=\infty^{2}\right)$. With this value of the critical size, the experimental time evolution of the resistivity is reasonably reproduced by cluster dynamics.

So as to better understand the evolution of the electrical resistivity we monitor the cluster size distribution ${ }^{3}$ and each cluster contribution to resistivity (Fig. 2) at different times of the precipitation kinetics for the supersaturation and the annealing temperature corresponding to Fig. 1. At the beginning of

$\overline{2}$ Cluster dynamics equations can be solved only for a finite number of classes. The infinite size in the simulations corresponds to a maximal size which can be as large as $\sim 10^{12}$ solute atoms. We check that the concentration of clusters having this maximal size does not evolve during the simulation. If this is not the case, the maximal size is increased.

3 Looking at the cluster size distribution, one should notice the abnormally high concentration of clusters containing 8 solute atoms. This is due to the low interface free energy associated with this size because of the existence of a compact cluster corresponding to a cube for this size [15].

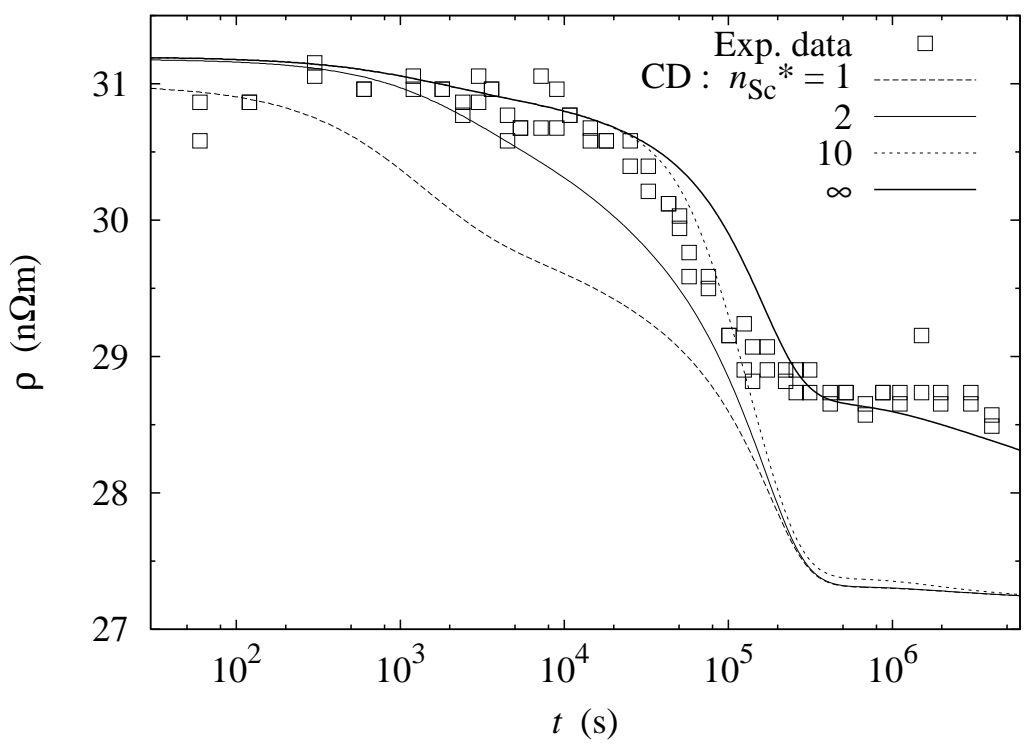

Fig. 1. Time evolution of the resistivity $\rho$ experimentally observed [10] and deduced from cluster dynamics simulations for a solid solution of composition $x_{\mathrm{Sc}}^{0}=0.12$ at. $\%$ annealed at $230^{\circ} \mathrm{C}$. Different critical size $n_{\mathrm{Sc}}^{*}$ have been used in cluster dynamics simulations (Eq. 1). 
the kinetics, $\left(t=10^{2}\right.$ or $\left.10^{4} \mathrm{~s}\right)$, only small clusters $\left(n_{\mathrm{Sc}} \leq 10\right)$ are present. Therefore, one can considers that resistivity only arises from these clusters. At the very beginning $\left(t \leq 10^{2} \mathrm{~s}\right)$, one can even only take into account monoand dimers. As precipitation goes on, the solid solution becomes depleted and thus the atomic fractions of small clusters decrease. For $t \geq 10^{6} \mathrm{~s}$, one can neglect the contributions of these small clusters to resistivity. On the contrary, as the precipitating phase appears, more and more large clusters are present in the system and only these large clusters contribute to the resistivity. For instance, for $t=10^{6} \mathrm{~s}$, almost all the resistivity is due to clusters containing between 10 and 50 solute atoms. Thus large clusters are responsible for the excess resistivity observed at the end of the precipitation kinetics. As we are

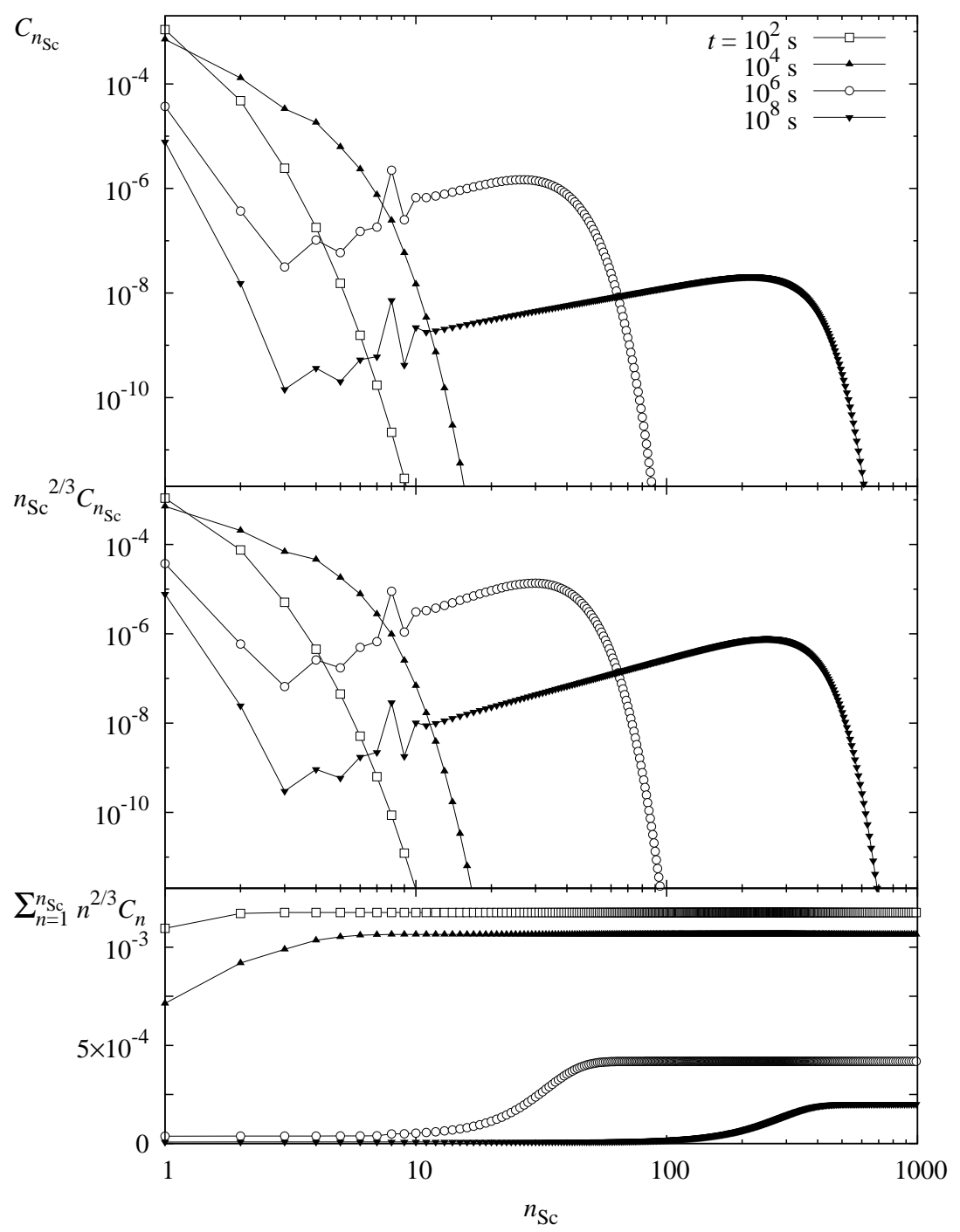

Fig. 2. Time evolution of the cluster size distribution $\left(C_{n_{\mathrm{Sc}}}\right)$, of the cluster contribution to electrical resistivity $\left(n_{\mathrm{Sc}}{ }^{2 / 3} C_{n_{\mathrm{Sc}}}\right)$ and of the cumulative contribution $\left(\sum_{n=1}^{n} C_{n} n^{2 / 3}\right)$ in a solid solution of composition $x_{\mathrm{Sc}}^{0}=0.12$ at. $\%$ annealed at $230^{\circ} \mathrm{C}$. Resistivities have been normalized by $\rho_{1}$. 
in the coarsening stage by this time, the concentrations of these larges clusters evolves very slowly, leading to a nearly stable resistivity.

\section{Comparison with experimental data}

Table 1

Summary of the experimental conditions (temperature, concentration $x_{\mathrm{Sc}}^{0}$, supersaturation $\left.x_{\mathrm{Sc}}^{0} / x_{\mathrm{Sc}}^{\mathrm{eq}}\right)$ corresponding to the available data used for the comparison with cluster dynamics simulations of the electrical resistivity variations in Al-Sc alloys.

\begin{tabular}{lccc}
\hline Reference & Temperature & Concentration & Supersaturation \\
\hline Røyset and Ryum [10] & $190^{\circ} \mathrm{C}$ & 0.12 at.\% & 3530 \\
& $230^{\circ} \mathrm{C}$ & 0.12 at.\% & 874 \\
& $270^{\circ} \mathrm{C}$ & 0.12 at.\% & 265 \\
& $330^{\circ} \mathrm{C}$ & 0.12 at.\% & 60 \\
& $250^{\circ} \mathrm{C}$ & 0.24 at.\% & 942 \\
Zakharov [8] & $300^{\circ} \mathrm{C}$ & 0.24 at.\% & 243 \\
& $350^{\circ} \mathrm{C}$ & 0.24 at.\% & 77.7 \\
& $400^{\circ} \mathrm{C}$ & 0.24 at.\% & 29.5 \\
& $400^{\circ} \mathrm{C}$ & 0.17 at.\% & 20.9 \\
& $450^{\circ} \mathrm{C}$ & 0.17 at.\% & 9.05 \\
Watanabe et al. [9] & $260^{\circ} \mathrm{C}$ & 0.09 at.\% & 264 \\
& $260^{\circ} \mathrm{C}$ & 0.15 at.\% & 440 \\
Jo and Fujikawa [6] & $300^{\circ} \mathrm{C}$ & 0.09 at.\% & 91 \\
& $300^{\circ} \mathrm{C}$ & 0.15 at.\% & 152 \\
& $370^{\circ} \mathrm{C}$ & 0.09 at.\% & 19.4 \\
& $370^{\circ} \mathrm{C}$ & 0.15 at.\% & 32.4 \\
\hline
\end{tabular}

We now compare cluster dynamics predictions with all the different experimental resistivity data available in the literature. All the experimental conditions corresponding to these data are given in Tab. 1. When doing this comparison, we use the critical size $n_{\mathrm{Sc}}=\infty$, i.e. we assume that all clusters contribute to the electrical resistivity. 


\section{$3.1 \mathrm{Al}-0.12$ at.\% Sc}

Røyset and Ryum [10] studied an alloy with a nominal composition ${ }^{4} x_{\mathrm{Sc}}^{0}=$ 0.12 at.\%. They followed the resistivity evolution during the precipitation kinetics for different annealing temperatures between 190 and $470^{\circ} \mathrm{C}$. All resistivity measurements were performed at room temperature for which the pure Al resistivity was measured to be $\rho_{\mathrm{Al}}^{0}=27.0 \mathrm{n} \Omega \mathrm{m}$. For temperatures lower than $330^{\circ} \mathrm{C}$, they observed that precipitates remain coherent and that precipitation is homogeneous. One can therefore compare their resistivity experimental data with cluster dynamics simulations (Fig. 3). A good agreement is obtained, especially for the lowest temperatures. Indeed, simulations manage to reproduce the resistivity fast decrease during the nucleation and growth stage as well as the slower variation which follows during coarsening. Nevertheless, for the highest temperature $\left(330^{\circ} \mathrm{C}\right)$ which corresponds to a lower supersaturation (Tab. 1), simulations appear to be too fast compared to experimental data. The shape of the curve is correct, but there is a timescale shift between the simulated and the experimental resistivity.

Røyset and Ryum [10] used their resistivity measurements to follow the Sc transformed fraction. In their treatment, they estimated the equilibrium solid solution from extrapolation of resistivity measurements in the coarsening stage

$\overline{4}$ They actually measured by EDS analysis a slightly higher composition, $x_{\mathrm{Sc}}^{0}=$ 0.138 at.\%, but cluster dynamics simulations obtained with this measured concentration do not significantly differ from those obtained with the nominal composition.

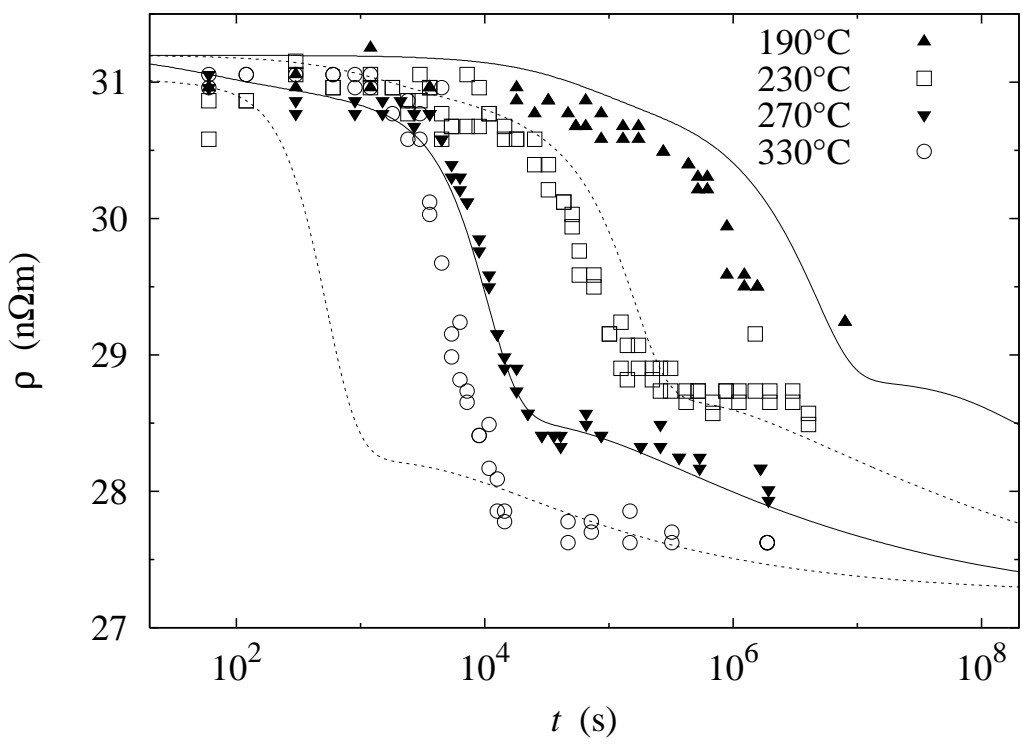

Fig. 3. Time evolution of the resistivity $\rho$ experimentally observed [10] and deduced from cluster dynamics simulations for a solid solution of composition $x_{\mathrm{Sc}}^{0}=0.12$ at. $\%$. 
to infinite time. They found that this method gave erroneous solvus estimates at low precipitation temperatures. Our simulations (Fig. 3) show that this is because of the excess resistivity associated with large clusters in the coarsening stage. For high annealing temperatures $\left(330^{\circ} \mathrm{C}\right.$ for instance), the measured resistivity is close to the equilibrium one, but for the lowest temperatures the difference cannot be neglected. When taking into account this excess resistivity one gets Sc transformed fractions different from those obtained by Røyset and Ryum (cf. appendix A). This explains why these authors deduced a wrong solubility limit for Sc in aluminum (Fig. 11 in Ref. 10), which they pointed out themselves, from their resistivity measurements. As cluster dynamics parameters were obtained so as to reproduce the experimental solubility limit [11] and as our simulations manage to reproduce Røyset and Ryum resistivity measurements, we can conclude that these measurements completely agree with the assessed Sc solubility limit [22].

\subsection{Al-0.24at.\% Sc}

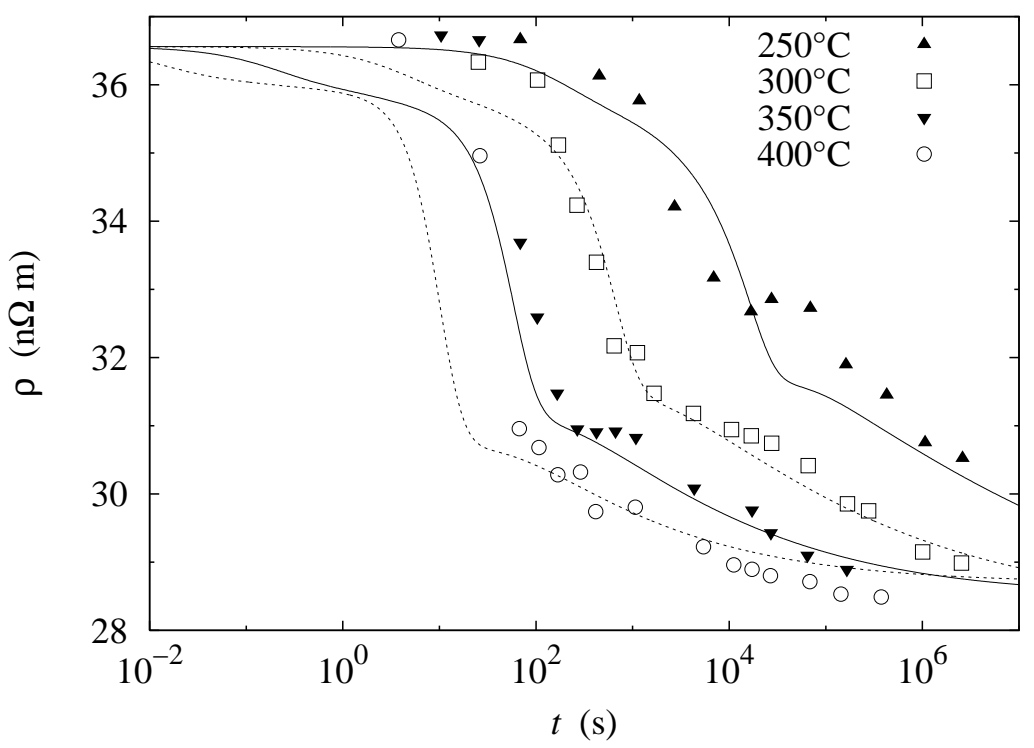

Fig. 4. Time evolution of the resistivity $\rho$ experimentally observed [8] and deduced from cluster dynamics simulations for a solid solution of composition $x_{\mathrm{Sc}}^{0}=0.24$ at. $\%$.

Zakharov [8] measured the electrical resistivity variations in an alloy containing 0.24 at.\% Sc annealed at different temperatures between 250 and $400^{\circ} \mathrm{C}$. He did not specify at which temperature he performed these measurements, nor the resistivity $\rho_{\mathrm{Al}}^{0}$ of pure Al. We assume in our simulations a resistivity $\rho_{\mathrm{Al}}^{0}=28.4 \mathrm{n} \Omega \mathrm{m}$, corresponding therefore to a higher measurement temperature or a lower aluminum purity than Røyset and Ryum measurements. 
Cluster dynamics reproduce quite well these experimental data (Fig. 4). For the lowest annealing temperatures $\left(250,300\right.$ and $\left.350^{\circ} \mathrm{C}\right)$, the agreement is perfect in all the different stages of the precipitation kinetics. For the highest temperature $\left(400^{\circ} \mathrm{C}\right)$, the simulated resistivity decreases too fast compared to the experimental one in the nucleation - growth stage. One should notice that the predicted evolution for this temperature is really fast and that the resistivity drop appears at a time $(t \sim 10 \mathrm{~s})$ which is too small to be precisely observed experimentally. Nevertheless, even for this temperature, simulations manage to reproduce the slow decreasing of electrical resistivity during coarsening.

\section{$3.3 \mathrm{Al}-0.17$ at.\% Sc}

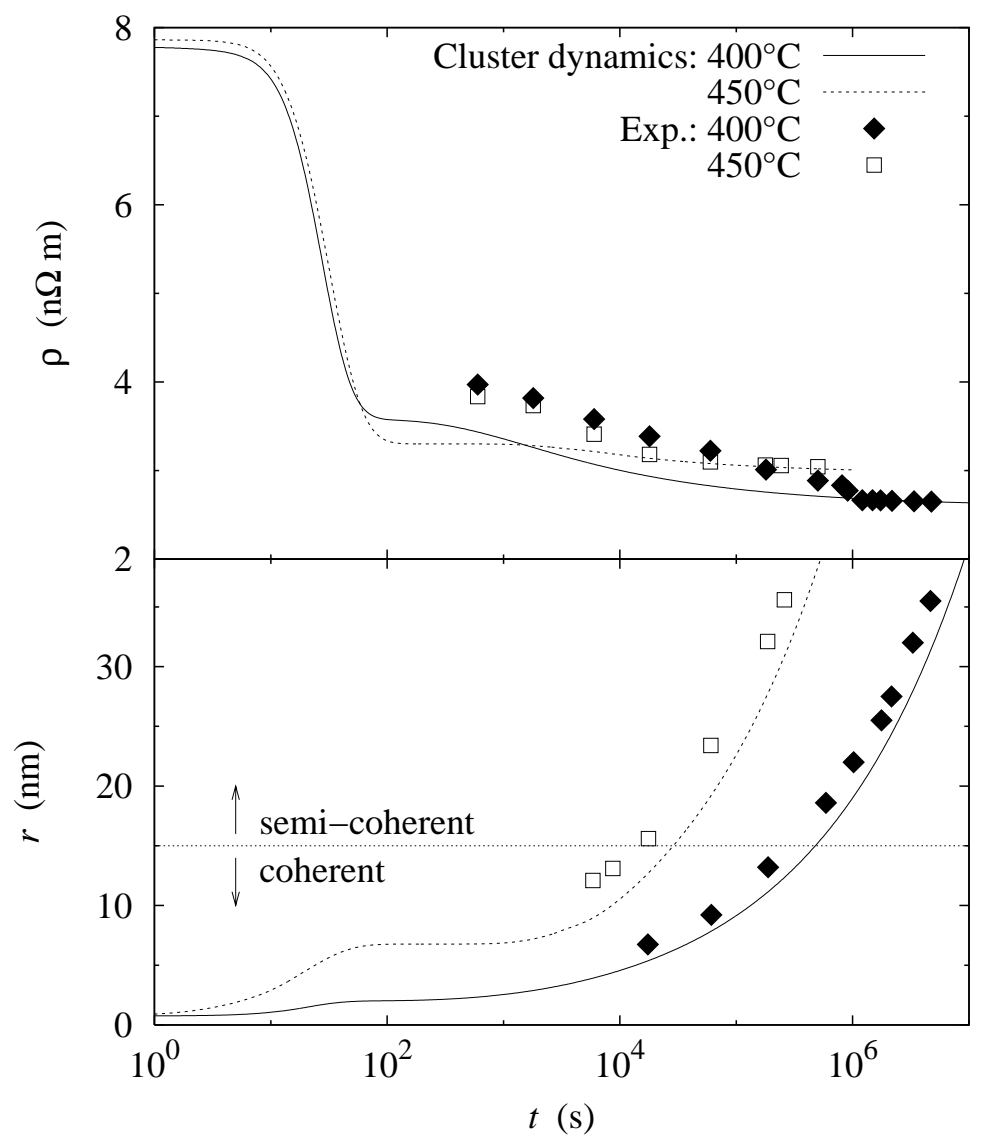

Fig. 5. Time evolution of the resistivity $\rho$ and of the mean precipitate radius $r$ experimentally observed [9] and deduced from cluster dynamics simulations for a solid solution of composition $x_{\mathrm{Sc}}^{0}=0.17$ at. $\%$. The cutoff radius used to define visible precipitates in cluster dynamics is $r^{*} \sim 0.75 \mathrm{~nm}$.

Watanabe et al. [9] studied an aluminum alloy with 0.17 at.\% Sc where they followed the resistivity during precipitation and measured the mean precipitate radius using transmission electron microscopy. All measurements were 
performed in the coarsening stage, after the resistivity had dropped because of precipitate nucleation and growth. As a consequence, only a partial comparison can be made with cluster dynamics (Fig. 5). The reasonable agreement obtained between the simulated and the experimental resistivities is not really significant as resistivity does not vary too much in the observed time range. Due to the high temperatures of study $\left(T \geq 400^{\circ} \mathrm{C}\right)$, the experimental as well as the simulated resistivities during coarsening are already close to that of the equilibrium solid solution.

Cluster dynamics manage to reproduce too the variations of the precipitate mean radius (Fig. 5). For radii greater than $\sim 15 \mathrm{~nm}$, Watanabe et al. observed that semi-coherent precipitates coexist with coherent ones. It should be pointed out that coherency loss is not taken into account in our simulations which handle only coherent precipitates. Despite this assumption, cluster dynamics predictions are not too bad. This may indicate that the fraction of semi-coherent precipitates is small or that these precipitates have an interface free energy not too far from the one of coherent precipitates.

\section{$3.4 A l-0.09$ at.\% Sc and $A l-0.15$ at.\% Sc}

Jo and Fujikawa [6] followed the resistivity variations in two different aluminum solid solutions containing 0.09 and 0.15 at.\% Sc. The simulated evolutions of the electrical resistivity do not reproduce well their measurements (Fig. 6). The resistivity drop associated with the nucleation and growth stage appears to occur too fast in simulations. Moreover, the slow decrease of the excess resistivity during coarsening is not as well reproduced as with other experimental data. Here, only a semi-quantitative agreement could be obtained.

\subsection{Summary}

Some more experimental data on resistivity measurements in Al-Sc alloys are available in the literature, but they cannot be used for a comparison with our simulations. Indeed, Nakayama et al. [7] studied an alloy containing 0.138 at.\% Sc aged at 250,300 and $350^{\circ} \mathrm{C}$. But they normalized their measurements and did not specify the measuring temperature nor the corresponding pure $\mathrm{Al}$ resistivity, thus forbidding any use of their data. Drits et al. [5] studied an alloy the Sc composition of which is larger than the Sc solubility limit and even the eutectic composition. Some primary $\mathrm{Al}_{3} \mathrm{Sc}$ precipitates may have appeared during the solidification and the alloy could not have been homogenized. Therefore, one can doubt that this alloy initial state corresponds to a homogeneous supersaturated solid solution as assumed by our simulations. Nevertheless, all the previously reviewed experimental data already allow to 


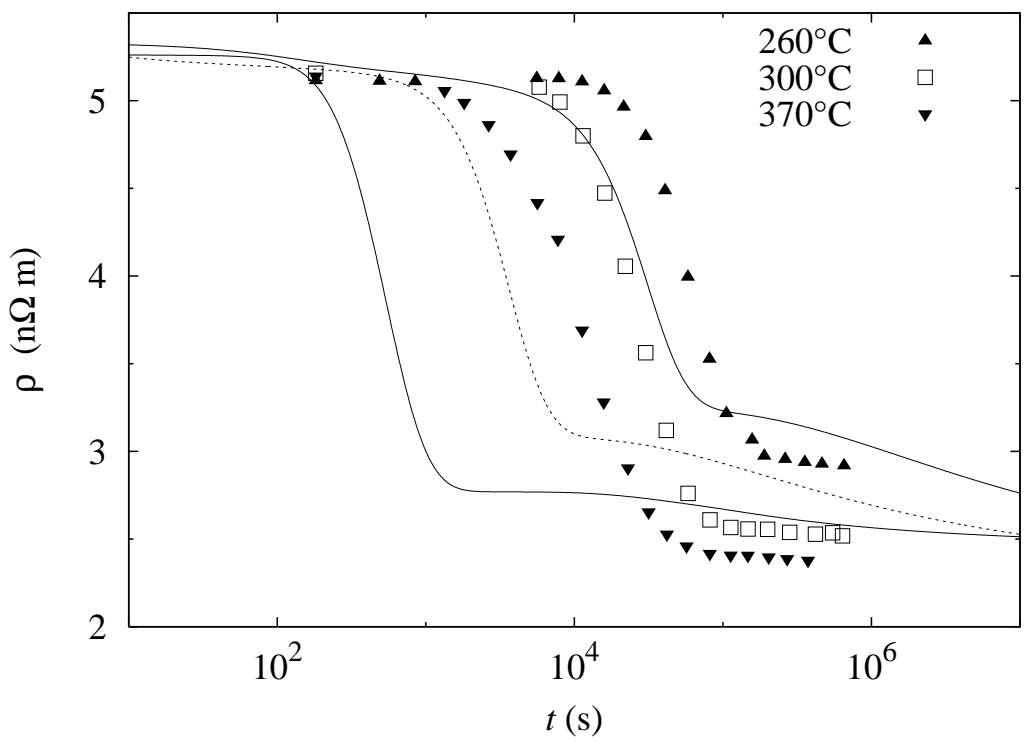

(a) $x_{\mathrm{Sc}}^{0}=0.09$ at. $\%$

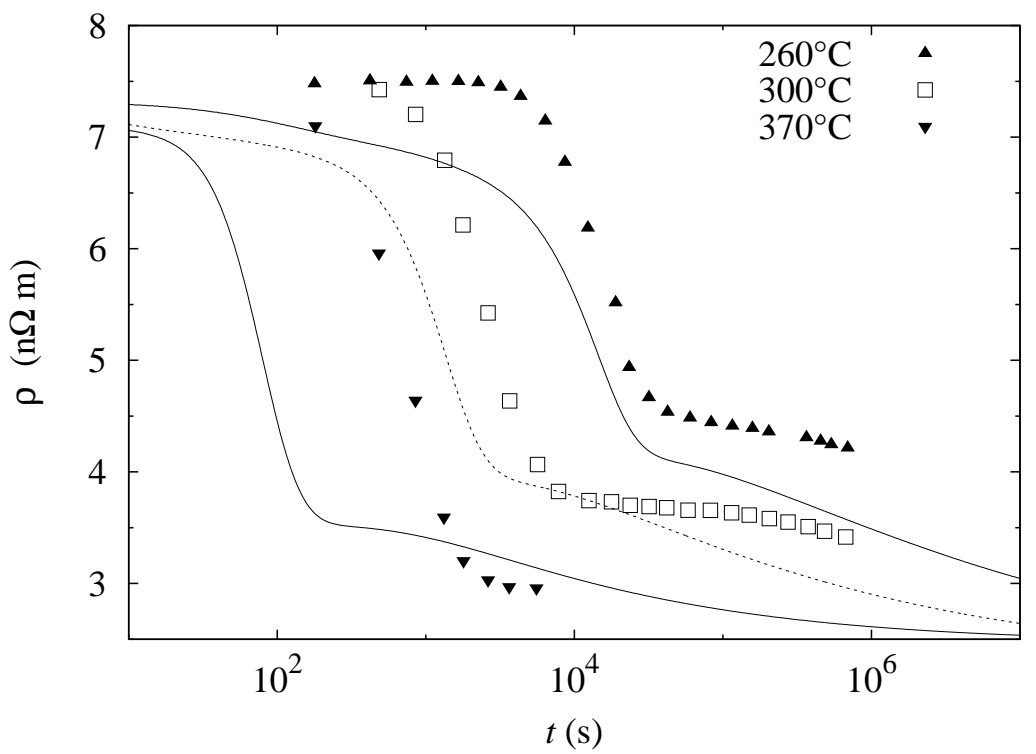

(b) $x_{\mathrm{Sc}}^{0}=0.15$ at. $\%$

Fig. 6. Time evolution of the resistivity $\rho$ experimentally observed [6] and deduced from cluster dynamics simulations for two solid solutions of composition $x_{\mathrm{Sc}}^{0}=0.09$ and 0.15 at.\%. 
draw main conclusions about the ability of cluster dynamics to predict electrical resistivity evolution during precipitation kinetics in Al-Sc alloys.

Except for Jo and Fujikawa's data [6] for which only a poor agreement could be obtained, cluster dynamics manage to reproduce reasonably well experimental measurements of electrical resistivity [8-10]. For the highest annealing temperatures corresponding to the lowest supersaturations, a time shift could appear between the simulated and the experimental resistivity, the time evolution predicted by cluster dynamics being too fast. Nevertheless, in all cases, the global shape of the evolution is correctly predicted. One can guess that a better description of the resistivity drop during precipitate nucleation and growth may be obtained if one would calculate precisely the small cluster contributions. But our simple model assuming a cluster contribution to the resistivity proportional to its area already leads to good predictions. Most importantly, cluster dynamics manage to reproduce quantitatively the slow resistivity decrease during coarsening. This decrease arises from the evolution of the large cluster distribution and the surface dependency assumption appears to be correct for these clusters.

An improvement of the agreement between cluster dynamics simulations and experimental data may arise too from a more precise calculation of the emission and condensation rate coefficients appearing in cluster dynamics master equation as suggested by Lépinoux $[23,24]$. But, doing so, we will lose one of the main advantages of our approach, i.e. the ease with which the parameters of the mesoscopic modeling can be deduced from a very limited number of input data. In view of its simplicity, the ability of our approach to reproduce resistivity measurements appears more than reasonable.

\section{Discussion}

Resistivity measurements are often combined with precipitate size determination using transmission microscopy so as to deduce from experimental data alloy parameters like the solute diffusion coefficient, the solubility limit, and the precipitate interface free energy. This can be done with the help of the LSW theory [2,3]. Indeed, Lifshitz and Slyozov [2] and Wagner [3] show that the precipitate mean radius varies linearly with the power $1 / 3$ of the time in the coarsening asymptotic limit. Ardell [4] then extended the theory to predict the variation of the solid solution concentration. Applying his results [25], the scandium concentration of the aluminum solid solution at time $t$ should be given by

$$
x_{\mathrm{Sc}}(t)=x_{\mathrm{Sc}}^{\mathrm{eq}}+(\kappa t)^{-1 / 3},
$$


where $x_{\mathrm{Sc}}^{\mathrm{eq}}$ is the scandium solubility in aluminum and the rate constant $\kappa$ is ${ }^{5}$

$$
\kappa=\frac{D_{\mathrm{Sc}}}{9}\left(\frac{k T}{x_{\mathrm{Sc}}^{\mathrm{eq}} \bar{\sigma} \Omega}\right)^{2} x_{\mathrm{Sc}}^{p} .
$$

$D_{\mathrm{Sc}}$ is the scandium impurity diffusion coefficient in aluminum, $x_{\mathrm{Sc}}^{p}=1 / 4$ is the Sc atomic fraction in the precipitate, $\bar{\sigma}$ is the infinite radius limit of the interface free energy between $\mathrm{Al}_{3}$ Sc precipitates and aluminum, and $\Omega=a^{3} / 4$ is the mean atomic volume corresponding to one lattice site $(a=4.032 \AA$ for $\mathrm{Al}$ ). Recently, Ardell and Ozolins [26] showed that the solute concentration can vary as the inverse square root of the time, and not the cube root like in Eq. 2, in the case where the precipitates present a ragged interface. This is not the case in Al-Sc alloys as the $\mathrm{Al}_{3} \mathrm{Sc}$ precipitate interfaces are rather sharp as shown by our atomic simulations $[15,16]$. Therefore, one expects Eq. 2 to hold for this system.

To make use of resistivity measurements, one usually assumes that resistivity depends linearly on the solid solution concentration. With the help of Eq. 2 and 3 , one can then get information on the desired parameters, i.e. $D_{\mathrm{Sc}}, x_{\mathrm{Sc}}^{\mathrm{eq}}$ or $\bar{\sigma}$. We previously saw that this linear relation between electrical resistivity and solute concentration does not hold in a phase-separating supersaturated Al-Sc solid solution as the resistivity has to be proportional to the cluster mean section (Eq. 1). It is worth looking at the error due to the fact that one identifies the solute concentration with the cluster mean section when exploiting resistivity measurements.

To do so, we need first to define the solid solution concentration in the cluster dynamics simulations. This is not so easy as this modeling technique describes the phase-separating alloy as a gas of solute clusters. Therefore, it does not differentiate between the solid solution and the precipitates at variance with precipitation classical descriptions like LSW theory (cf. Ref. 27 for a better understanding of the differences between cluster dynamics and classical theories). Nevertheless, one can discriminate the solid solution and the precipitates with the help of a threshold size $n_{\mathrm{Sc}}^{\text {th }}$. Below this size, clusters represents fluctuations in the solid solution and above it they represent stable precipitates. The solid solution concentration is thus given by

$$
x_{\mathrm{Sc}}(t)=\sum_{n_{\mathrm{Sc}}=1}^{n_{\mathrm{Sc}}^{\mathrm{th}}} n_{\mathrm{Sc}} C_{n_{\mathrm{Sc}}}(t)
$$

In a supersaturated solid solution, one natural choice for this threshold size is the critical size $n_{\mathrm{Sc}}^{*}$. In cluster dynamics, this is the size for which the

\footnotetext{
5 When using results obtained by Calderon et al. [25] for non pure precipitates, we assume an ideal solid solution and we use the fact that $x_{\mathrm{Sc}}^{\mathrm{eq}}<<x_{\mathrm{Sc}}^{p}$.
} 
condensation rate $\beta_{n_{\mathrm{Sc}}}$ is equal to the emission rate $\alpha_{n_{\mathrm{Sc}}}$. Below this size, $\beta_{n_{\mathrm{Sc}}}$ is smaller than $\alpha_{n_{\mathrm{Sc}}}$ and clusters have more chance to re-dissolve themselves than to grow. This definition works as long as we do not enter in the coarsening stage. A minimum for a size $n_{\mathrm{Sc}}^{\min }$ then appears in the cluster size distribution $\left(n_{\mathrm{Sc}}^{\min }=3\right.$ for $t=10^{6}$ and $10^{8} \mathrm{~s}$ in Fig. 2). Once the critical size $n_{\mathrm{Sc}}^{*}$ gets higher than $n_{\mathrm{Sc}}^{\min }$, the quantity of matter contained in clusters smaller than $n_{\mathrm{Sc}}^{*}$ begins to increase artificially because of small precipitates which become unstable. We then choose the following definition for the threshold size:

- $n_{\mathrm{Sc}}^{\mathrm{th}}=n_{\mathrm{Sc}}^{*}$, as long as the cluster size distribution does not show any minimum,

- $n_{\mathrm{Sc}}^{\mathrm{th}}=\min \left(n_{\mathrm{Sc}}^{*}, n_{\mathrm{Sc}}^{\min }\right)$ otherwise.

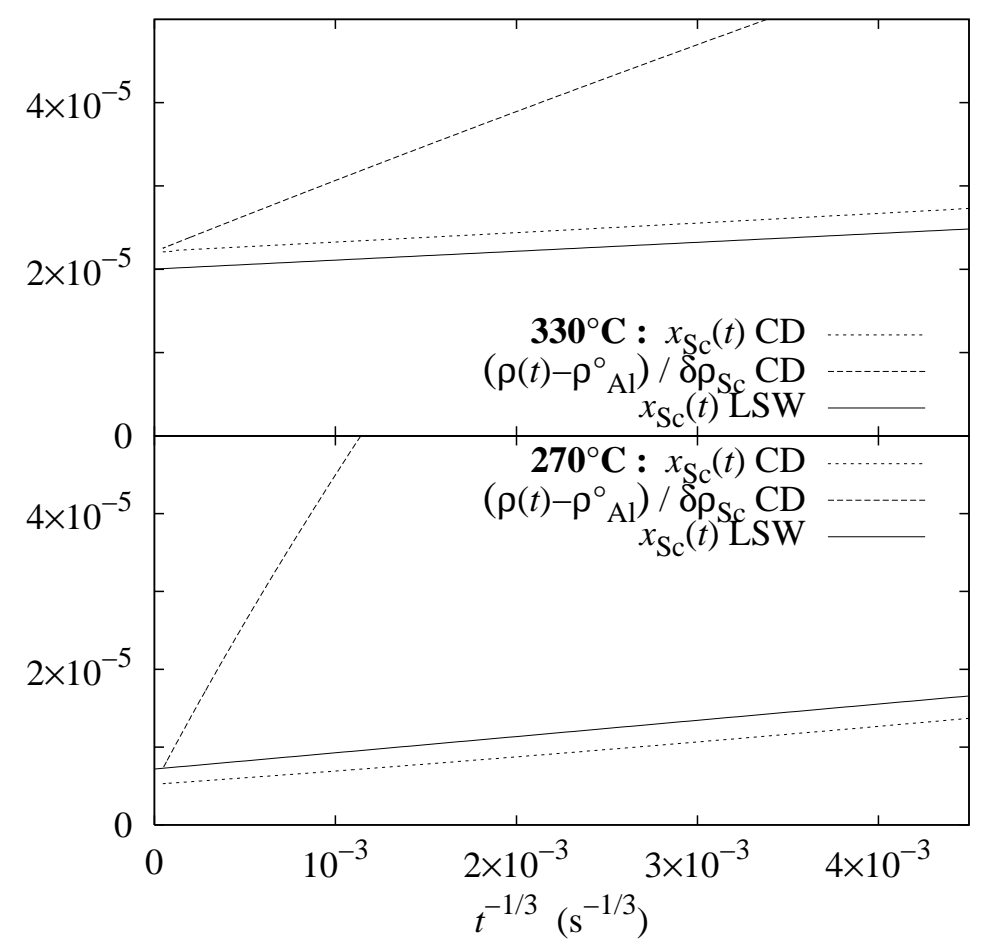

Fig. 7. Change as a function of $t^{-1 / 3}$ in the Sc concentration $x_{\mathrm{Sc}}(t)$ (Eq. 4) and in the normalized resistivity $\left(\rho(t)-\rho_{\mathrm{Al}}^{0}\right) / \delta \rho_{\mathrm{Sc}}$ (Eq. 1) simulated by cluster dynamics in an aluminum solid solution containing 0.12 at. $\%$ Sc annealed at $270^{\circ} \mathrm{C}$ and $330^{\circ} \mathrm{C}$. The linear relation predicted by LSW theory (Eq. 2) is shown for comparison.

Using this definition of the threshold size, we monitor the variations of the solid solution concentration (Eq. 4) in clusters dynamics simulations for the annealing at 270 and $330^{\circ} \mathrm{C}$ of a solid solution containing 0.12 at.\% Sc (Fig. 7). The asymptotic behavior of $x_{\mathrm{Sc}}$ clearly obeys a linear dependence with $t^{-1 / 3}$ as predicted by LSW theory. Using the solid solubility $x_{\mathrm{Sc}}^{\mathrm{eq}}$, the diffusion coefficient $D_{\mathrm{Sc}}$ and the interface free energy $\bar{\sigma}$ which the simulations rely on (cf. Ref. 15 and 11 for a full description of the way clusters dynamics parameters were obtained), we can calculate coefficients entering Eq. 2. The comparison 
with cluster dynamics simulations (Fig. 7) shows that LSW perfectly manages to predict the asymptotic behavior of the solid solution concentration $x_{\mathrm{Sc}}(t)$. On the other hand, Fig. 7 shows that the asymptotic behavior of the normalized resistivity $\left(\rho(t)-\rho_{\mathrm{Al}}^{0}\right) / \delta \rho_{\mathrm{Sc}}$ differs from the one of the solid solution concentration. Both quantities tend to a limit close to the scandium solubility $x_{\mathrm{Sc}}^{\mathrm{eq}}$ in aluminum, but the resistivity cannot be assumed to vary linearly with $t^{-1 / 3}$. If one does so, the proportionality coefficient will differ from the one predicted by LSW theory (Eq. 3). As a consequence, if one assumes that the resistivity is proportional to the solid solution concentration and uses LSW theory to deduce alloy properties from experimental data, the obtained solid solubility will be correct, but not the proportionality coefficient $\kappa$. In particular, one cannot deduce from resistivity measurements any reliable value for the precipitate interface free energy $\bar{\sigma}$ or the solute diffusion coefficient $D_{\mathrm{Sc}}$. The lower the annealing temperature, the bigger the error on these parameters. For instance, fitting with Eq. 2 and 3 the normalized resistivity evolution in Fig. 7, one would get $\bar{\sigma}=2200$ instead of $113 \mathrm{~mJ} . \mathrm{m}^{-2}$ at $T=330^{\circ} \mathrm{C}$. This clearly illustrates that resistivity measurements cannot be used to determine interface free energy in a phase-separating alloy as long as precipitates contribute to resistivity.

Of course, this conclusion does not hold anymore when this precipitate contribution cancels. Indeed Watanabe et al. [9] manages to observe a linear variation of the resistivity with $t^{-1 / 3}$. This behavior was obtained once the precipitates became incoherent. Therefore, one can reasonably assume that incoherent $\mathrm{Al}_{3} \mathrm{Sc}$ precipitates do not contribute anymore to resistivity. The interface free energies they deduced from their resistivity measurements $\left(230 \mathrm{~mJ} . \mathrm{m}^{-2}\right.$ between 400 and $450^{\circ} \mathrm{C}$ ) is higher than the one used in our simulations (119 $\geq$ $\bar{\sigma} \geq 105 \mathrm{~mJ} . \mathrm{m}^{-2}$ between 200 and $500^{\circ} \mathrm{C}$ ) which was deduced from the atomic diffusion model of Ref. 15,18. This is in agreement with the fact that incoherent precipitates should have a higher interface free energy than coherent ones.

\section{Conclusions}

Cluster dynamics has been shown to be able to reproduce electrical resistivity variations during precipitation in Al-Sc alloys. One should recall that the only input parameters required by this modeling technique are the solute diffusion coefficients, the precipitate/aluminum interface free energy and the resistivity increase per solute atom. None of these parameters was adjusted to fit the experimental data. Indeed, the experimental data measured by Fujikawa et al. $[6,21]$ was used for the resistivity increase with solute content $\left(\delta \rho_{\mathrm{Sc}}=\right.$ $3400 \mathrm{n} \Omega \mathrm{m}$ ). Good predictions were obtained with this experimental value. As for the solute diffusion coefficients and the interface free energies, they 
were directly deduced [11] from an atomic model previously developed for Al-Zr-Sc alloys $[15,18]$. The good agreement obtained between our resistivity simulations and experimental data allows then us to stress the correctness of the $\mathrm{Al}_{3} \mathrm{Sc}$ interface free energies used in our simulations. For temperatures ranging between 200 and $500^{\circ} \mathrm{C}$, this interface free energy corresponding to coherent $\mathrm{Al}_{3} \mathrm{Sc}$ precipitates is varying between 119 and $105 \mathrm{~mJ} . \mathrm{m}^{-2}$.

One of the key assumptions of our simulations is that all clusters contribute to electrical resistivity and that each cluster contribution is proportional to its area. Although this assumption may look crude, it leads to quantitative predictions. In particular, it manages to reproduce the resistivity excess and its slow decrease during coarsening. This excess resistivity mainly arises from large clusters contributions whereas the solid solution contribution can be neglected. As a consequence, resistivity measurements during coarsening do not really allow to follow the solid solution concentration. In particular, resistivity do not obey LSW theory in a phase-separating system like supersaturated AlSc alloys at variance with the solid solution concentration. This involves that one cannot deduce from these measurements correct values of the precipitate interface free energy or of the solute diffusion coefficients. On the other hand, solubility limits obtained from resistivity experiments are correct as both the normalized resistivity and the solid solution concentration tend to the same value for long enough annealing times.

\section{A Reinterpretation of Røyset experimental data}

Usually, one uses electrical resistivity measurements to define the fraction of precipitated solute

$$
X_{\rho}(t)=\frac{\rho(t)-\rho^{0}}{\rho^{\mathrm{eq}}-\rho^{0}}
$$

where $\rho(t)$ is the resistivity of the solid solution measured at time $t$ and $\rho^{0}$ and $\rho^{\text {eq }}$ are respectively the initial and equilibrium resistivities. If the resistivity was truly proportional to the solute concentration like it can be assumed in an under-saturated solid solution, this definition would be equivalent to the one obtained from considering the solid solution concentration instead of the resistivity. But in a phase-separating system like supersaturated Al-Sc solid solution, both definitions lead to different quantities.

In their work [10], Røyset and Ryum used Eq. A.1 to define the precipitated fraction. Assuming that resistivity varies linearly with $t^{-1 / 3}$ in the coarsening stage, extrapolation of data to infinite time was used to estimate the equilibrium resistivity $\rho^{\mathrm{eq}}$. This leads to a correct value of $\rho^{\mathrm{eq}}$ for the higher annealing temperatures but not for the lower ones $\left(T \leq 330^{\circ} \mathrm{C}\right)$. Indeed, as we previously saw, one cannot assume that resistivity follows the same time dependence as 

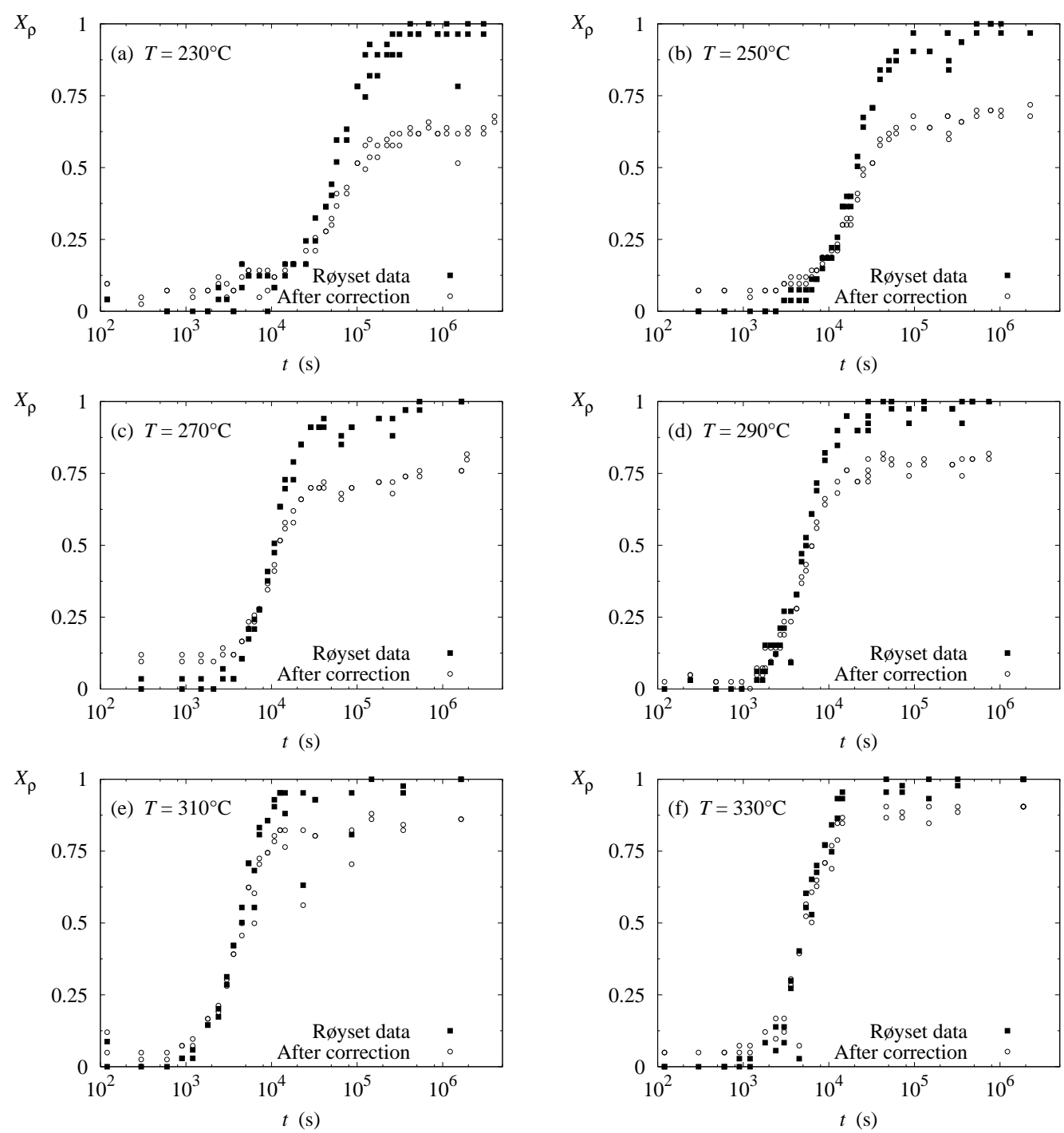

Fig. A.1. Evolution with time of the precipitated fraction $X_{\rho}$ in an aluminum solid solution of composition $x_{\mathrm{Sc}}^{0}=0.12$ at. $\%$. $X_{\rho}$ has been deduced from experimental resistivity measurements [10] considering the equilibrium resistivity is reached (Røyset data) or not (after correction) for the longest annealing time.

the solid solution concentration due to the large cluster contributions to electrical resistivity. Therefore $\rho^{\mathrm{eq}}$ cannot be obtained from such an extrapolation to infinite time, and it has to be calculated from the equilibrium Sc solubility in aluminum and the linear relation $[6,21]$ between resistivity and concentration existing in dilute solid solutions leading to

$$
\rho^{\mathrm{eq}}=\rho_{\mathrm{Al}}^{0}+\delta \rho_{\mathrm{Sc}} x_{\mathrm{Sc}}^{\mathrm{eq}},
$$

The precipitated fraction $X_{\rho}$ obtained calculating $\rho^{\mathrm{eq}}$ in this way differs from the one obtained by Røyset and Ryum who assumed that $\rho^{\mathrm{eq}}$ corresponds to the resistivity measurements for the longest annealing time (Fig. A.1). This is a clear manifestation of the large cluster contributions to electrical resistivity. 


\section{Acknowledgements}

The authors are grateful to Dr. Røyset and Dr. Watanabe for providing experimental data. They want to thank too Dr. Martin and Dr. Soisson for their careful reading of the manuscript, as well as Dr. Limoge, Dr. Marinica and Dr. Proville for useful discussions on electron scattering.

\section{References}

[1] P. L. Rossiter, The Electrical Resistivity of Metals and Alloys, Cambridge Universtiy Press, Cambridge, 1987.

[2] I. M. Lifshitz, V. V. Slyozov, J. Phys. Chem. Solids 19 (1961) 35.

[3] C. Wagner, Z. Elektrochem. 65 (1961) 581.

[4] A. J. Ardell, Acta Metall. 15 (1967) 1772.

[5] M. E. Drits, J. Dutkiewicz, L. S. Toropova, J. Salawa, Crystal Res. Technol. 19 (1984) 1325.

[6] H.-H. Jo, S.-I. Fujikawa, Mater. Sci. Eng. A 171 (1993) 151.

[7] M. Nakayama, A. Furuta, Y. Miura, Mater. T. JIM 38 (10) (1997) 852.

[8] V. V. Zakharov, Metal Science and Heat Treatment 39 (1997) 61.

[9] C. Watanabe, T. Kondo, R. Monzen, Metall. Mater. Trans. A 35 (2004) 3003.

[10] J. Røyset, N. Ryum, Mater. Sci. Eng. A 396 (2005) 409.

[11] E. Clouet, A. Barbu, L. Laé, G. Martin, Acta Mater. 53 (2005) 2313.

[12] G. M. Novotny, A. J. Ardell, Mater. Sci. Eng. A 318 (2001) 144.

[13] E. A. Marquis, D. N. Seidman, Acta Mater. 49 (2001) 1909.

[14] E. A. Marquis, D. N. Seidman, D. C. Dunand, Acta Mater. 50 (2002) 4021.

[15] E. Clouet, M. Nastar, C. Sigli, Phys. Rev. B 69 (2004) 064109.

[16] E. Clouet, Séparation de phase dans les alliages Al-Zr-Sc: du saut des atomes à la croissance de précipités ordonnés, Ph.D. thesis, École Centrale Paris (2004). URL http://tel.ccsd.cnrs.fr/tel-00005967

[17] E. Clouet, M. Nastar, A. Barbu, C. Sigli, G. Martin, in: J. M. Howe, D. E. Laughlin, J. K. Lee, U. Dahmen, W. A. Soffa (Eds.), Solid-Solid Phase Transformations in Inorganic Materials, Vol. 2, TMS, 2005, p. 683.

[18] E. Clouet, L. Laé, T. Épicier, W. Lefebvre, M. Nastar, A. Deschamps, Nat. Mater. 5 (2006) 482. 
[19] C.-C. Fu, J. Dalla Torre, F. Willaime, J.-L. Bocquet, A. Barbu, Nat. Mater. 4 (2005) 68.

[20] J. Merlin, G. Vigier, Phys. Status Solidi A 58 (1980) 571.

[21] S. I. Fujikawa, M. Sugaya, H. Takei, K. I. Hirano, J. Less-Common Met. 63 (1979) 87.

[22] J. Murray, J. Phase Equilib. 19 (1998) 380.

[23] J. Lépinoux, Philos. Mag. 85 (2005) 3585.

[24] J. Lépinoux, Philos. Mag. 86 (2006) 5053.

[25] H. A. Calderon, P. W. Voorhees, J. L. Murray, G. Kostorz, Acta Metall. Mater. 42 (1994) 991.

[26] A. J. Ardell, V. Ozolins, Nat. Mater. 4 (2005) 309.

[27] G. Martin, in: J. M. Howe, D. E. Laughlin, J. K. Lee, U. Dahmen, W. A. Soffa (Eds.), Solid-Solid Phase Transformations in Inorganic Materials, Vol. 2, TMS, 2005, p. 291. 